\title{
The fate of LSB galaxies in clusters and the origin of the diffuse intra-cluster light
}

\author{
Ben Moore \\ Department of Physics, University of Durham, South Road, Durham, \\ DH1 3LE, UK \\ George Lake, Joachim Stadel \& Thomas Quinn \\ Department of Astronomy, University of Washington, Seattle, \\ WA98195, USA
}

\begin{abstract}
.
We follow the evolution of disk galaxies within a cluster that forms hierarchically in a standard cold dark matter $\mathrm{N}$-body simulation. At a redshift $z=0.5$ we select several dark matter halos that have quiet merger histories and are about to enter the newly forming cluster environment. The halos are replaced with equilibrium high resolution model spirals that are constructed to represent luminous examples of low surface brightness (LSB) and high surface brightness (HSB) galaxies. Whilst the models have the same total luminosity, $\sim L_{*}$, they have very different internal mass profiles, core radii and disk scale lengths, however they all lie at the same place on the Tully-Fisher relation. Due to their "soft" central potentials, LSB galaxies evolve dramatically under the influence of rapid encounters with substructure and strong tidal shocks from the global cluster potential - galaxy harassment. As much as $90 \%$ of the LSB disk stars are tidally stripped and congregate in large diffuse tails that trace the orbital path of the galaxy and form the diffuse intra-cluster light. The bound stellar remnants closely resemble the dwarf spheroidals (dE's) that populate nearby clusters, with large scale lengths and low central surface brightness.
\end{abstract}

\section{Introduction}

Clusters of galaxies provide a unique environment wherein the galaxy population has been observed to rapidly evolve over the past few billion years (Butcher \& Oemler 1978, Dressler et al. 1998). At a redshift $z \geq 0.4$, clusters are dominated by spiral galaxies that are predominantly faint irregular or Sc-Sd types. Some of these spirals have disturbed morphologies; many have high rates of star-formation (Dressler et al. 1994a). Conversely, nearby clusters are almost completely dominated by spheroidal (dSph), lenticulars (S0) and elliptical galaxies (Bingelli et al. 1987, 1988, Thompson \& Gregory 1993). Observations suggest that the elliptical galaxy population was already in place at much higher redshifts, at which time the SO population in clusters is deficient compared to 
nearby clusters (Couch et al. 1998, Dressler et al. 1998). This evolution of the morphology-density relation appears to be driven by an increase in the S0 fraction with time and a corresponding decrease in the luminous spiral population.

Low surface brightness (LSB) galaxies appear to avoid regions of high galaxy densities (Bothun et al. 1993, Mo et al. 1994). This is somewhat puzzling since recent work by Mihos et al. (1997) demonstrated that LSB disk galaxies are actually more stable to close tidal encounters than HSB disk galaxies. In fact, LSB galaxies have lower disk mass surface densities and higher mass-to-light ratios, therefore their disks are less susceptible to internal global instabilities, such as bar formation. However, in a galaxy cluster, encounters occur frequently and very rapidly, on a shorter timescale than investigated by Mihos et al. and the magnitude of the tidal shocks are potentially very large.

Several physical mechanisms have been proposed that can strongly affect the morphological evolution of disks: ram-pressure stripping (Gunn \& Gott 1978), galaxy merging (Icke 1985, Lavery \& Henry 1988, 1994) and galaxy harassment (Moore et al. 1996a, 1998). The importance of these mechanisms varies with environment: mergers are frequent in groups but rare in clusters (Ghigna et al. 1998), ram pressure removal of gas is inevitable in rich clusters but will not alter disk morphology (Abadi \& Moore, in preparation). The morphological transformation in the dwarf galaxy populations $\left(M_{b}>-16\right)$ in clusters since $z=0.4$ can be explained by rapid gravitational encounters between galaxies and accreting substructure - galaxy harassment. The impulsive and resonant heating from rapid fly-by interactions causes a transformation from disks to spheroidals.

The numerical simulations of Moore et al. focussed on the evolution of fainter Sc-Sd spirals in static cluster-like potentials and their transition into dSph's. In this work we shall examine the role of gravitational interactions in driving the evolution of luminous spirals in dense environments. We will use more realistic simulations that follow the formation and growth of a large cluster that is selected from a cosmological simulation of a closed CDM universe. The parameter space for the cluster model is fairly well constrained once we have adopted hierarchical structure formation. The structure and substructure of virialised clusters is nearly independent of the shape and normalisation of the power spectrum. Clusters that collapse in low Omega universes form earlier, thus their galaxies have undergone more interactions. The cluster that we follow virialises at $z \sim 0.3$, leaving about 4 Gyrs for the cluster galaxies to evolve.

The parameter space for the model spirals is much larger. Mihos et al. examined the effects of a single encounter at a fixed number of disk scale lengths, whilst varying the disk surface brightness and keeping other properties fixed. The key parameter that determines whether or not dark matter halos survive within a cluster $\mathrm{N}$-body simulation is the core radius of the substructure, which is typically dictated by the softening length (Moore, Katz \& Lake 1996b). We suspect that the "softness" of the dark matter potentials may also be the key factor that governs whether or not a given disk galaxy will survive within a real cluster. 


\section{The model galaxies}

We use the technique developed by Hernquist (1989) to construct equilibrium spiral galaxies with disk, bulge and halo components, that represent "standard" HSB and LSB disk galaxies. In each model the disk mass is $4.0 \times 10^{10} M_{\odot}$ and the rotation curves both peak at $200 \mathrm{~km} \mathrm{~s}^{-1}$. They are a little less massive than " $L_{*}$ " galaxies, the characteristic luminosity of the break in the galaxy luminosity function and would have absolute magnitudes $\sim-17.8$ for a mass to light ratio of 5. The "HSB" spiral has an exponential disk scale length, $r_{d}=3.0 \mathrm{kpc}$ and a bulge with a mass of one third of the total disk mass. The "LSB" disk scale length is $10 \mathrm{kpc}$ and has no bulge. The scale height, $r_{z}$, of each disk is $0.1 r_{d}$ and they are constructed with a Toomre $Q$ parameter of 1.5. Each galaxy has a dark halo modeled by truncated isothermal spheres with core radii set equal to the disk scale length. This scaling ensures that each galaxy lies at the same point on the Tully-Fisher relation, yet the galaxies will have different internal mass distributions (Zwaan et al. 1995).
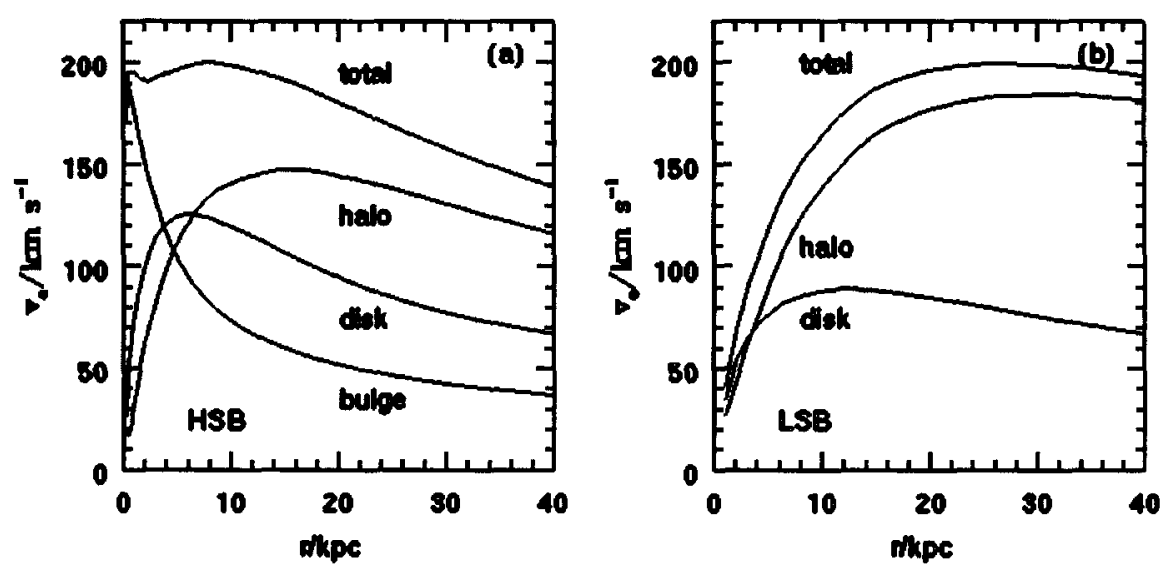

Figure 1. The curves show the contributions from stars and dark matter to the total rotational velocity of the disk within (a) the HSB galaxy and (b) the $L S B$ galaxy.

Figure 1 shows the contribution to the rotation velocity of the disks from each component. Note that the bulge component of the HSB galaxy has ensured that the rotation curve is close to flat over the inner 5 disk scale lengths, whereas the rotation curve of the LSB galaxy rises slowly over this region. These rotation curves are typical of that measured for LSB galaxies (de Blok \& McGaugh 1996) and HSB galaxies (Persic \& Salucci 1997).

Each disk is modeled using 20,000 star particles of mass $2 \times 10^{6} M_{\odot}$ and 40,000 dark matter halo particles of mass $2 \times 10^{7} M_{\odot}$ in the LSB and $6 \times 10^{6} M_{\odot}$ in the HSB galaxy. The force softening is $0.1 r_{d}$ for the star particles and $0.5 r_{d}$ for the halo particles. Their disks are stable and they remain in equilibrium when simulated in isolation. Discreteness in the halo particles causes the disk scale height to increase with time as quantified in Section 4 for the LSB galaxy. 


\section{The response to impulsive encounters}

For a given orbit through a cluster, the visible response of a disk galaxy to a tidal encounter depends primarily upon its internal dynamical timescale. Galaxies with cuspy central mass distributions, such as ellipticals, have short orbital timescales at their centres and they will respond adiabatically to tidal perturbations. Sa-Sb spirals have flat rotation curves, therefore a tidal encounter will cause an impulsive disturbance to a distance $\sim v_{c} b / V$, where $b$ is the impact parameter, $V$ is the encounter velocity and $v_{c}$ is the galaxy's rotation speed. LSB galaxies and Sc-Sd galaxies have slowly rising rotation curves, indicating that the central regions are close to a uniform density. The central dynamical timescales are constant throughout the inner disk and an encounter that is impulsive at the core radius will be impulsive throughout the galaxy.

The strength of an encounter is $\propto M_{p}^{2} / V^{2}$, where $M_{p}$ is the perturbing mass. The typical galaxy-galaxy encounter within a virialised cluster occurs at a relative velocity $\sim \sqrt{2} \sigma_{1 d}$. Substituting typical parameters for an Sa-Sb spiral orbiting within a cluster, we find that such encounters will not perturb the disk within $\sim 3 r_{d}$. However, tidal shocks from the mean cluster field also provides a significant heating source for those galaxies on eccentric orbits (Byrd \& Valtonen 1990, Valluri 1993). Ghigna et al. (1998) studied the orbits of several hundred dark halos within a cluster that formed hierarchically in a cold dark matter universe. The median ratio of apocenter to pericenter was $6: 1$, with a distribution skewed towards radial orbits. More than $20 \%$ of the halos were on orbits more radial than 10:1. A galaxy on this orbit would move past pericenter at several thousand $\mathrm{km} \mathrm{s}^{-1}$ and would be heated across the entire disk.

We illustrate the effect of a single impulsive encounter on each of our model disks in Figure 2 and Figure 3. At time $\mathrm{t}=0$ we send a perturbing halo of mass $2 \times 10^{12} M_{\odot}$ perpendicular to the plane of the disk at an impact parameter of $60 \mathrm{kpc}$ and velocity of $1500 \mathrm{~km} \mathrm{~s}^{-1}$. This encounter would be typical of that occurring in a rich cluster with a tidally truncated $L_{*}$ elliptical galaxy near the cluster core. Any one galaxy in the cluster will suffer several encounters stronger than this since the cluster formed. Although we simulate a perpendicular orbit here, we do not expect the encounter geometry to make a significant difference since the difference between direct and retrograde encounters will be small i.e. $V>v_{c}$.

At $t=0.1$ Gyrs after the encounter, the perturber has moved $150 \mathrm{kpc}$ away, yet the visible response to the encounter is hardly apparent. After $0.2 \mathrm{Gyrs}$, we can begin to see the response to the tidal shock as material is torn from the disk into extended tidal arms. Even at this epoch their is a clear difference to the response of the perturbation by each galaxy. After 0.4 Gyrs, the LSB galaxy is dramatically altered over the entire disk and a substantial fraction of material has been removed past the tidal radius. Remarkably, the central disk of the HSB galaxy remains intact and only the outermost stars have been strongly perturbed. 


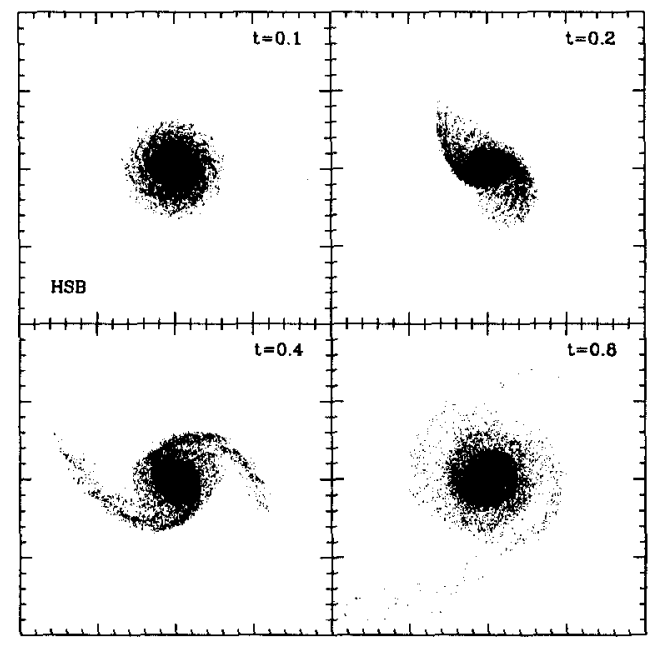

Figure 3. Snapshots of the distribution of disk stars from a HSB galaxy after a single high-speed encounter with a massive galaxy. Each frame is 120 kpc on a side and encounter takes place perpendicular to the disk at the box edge $(60 \mathrm{kpc})$.

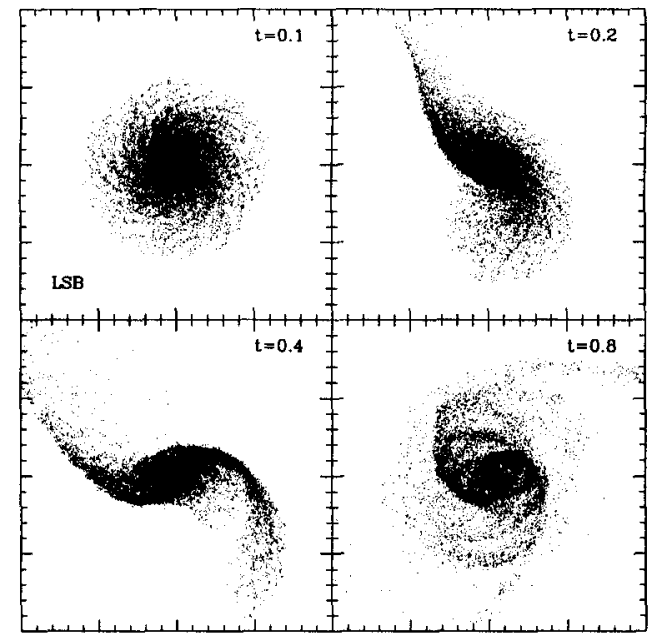

Figure 4. Snapshots of the distribution of disk stars from an LSB galaxy after a single high-speed encounter with a massive galaxy. Each frame is 120 $k p c$ on a side and the encounter takes place perpendicular to the disk at the box edge $(60 \mathrm{kpc})$. 


\section{Simulating disk evolution within a hierarchical universe}

Previous simulations of tidal shocks and galaxy harassment focussed upon the evolution of disk galaxies in static clusters with substructure represented by softened potentials with masses drawn from a Schechter function (Moore $e t$ al. 1996 a \& 1998). Here we use a more realistic approach of treating the perturbations by following the growth of a cluster within a hierarchical cosmological model. The cluster was extracted from a large CDM simulation of a closed universe within a $50 \mathrm{Mpc}$ box and was chosen to be virialised by the present epoch. (We assume a Hubble constant of $100 \mathrm{~km} \mathrm{~s}^{-1} \mathrm{Mpc}^{-1}$.) Within the turn-around region there are $\sim 10^{5} \mathrm{CDM}$ particles of mass $10^{10} M_{\odot}$ and their softening length is $20 \mathrm{kpc}$. At a redshift $z=0$ the cluster has a one dimensional velocity dispersion of $700 \mathrm{~km} \mathrm{~s}^{-1}$ and a virial radius of $2 \mathrm{~h}^{-1} \mathrm{Mpc}$. The tidal field from the mass distribution beyond the cluster's turn-around radius is simulated with massive particles to speed the computation.

Between a redshift $z=2$ to $z=0.5$ we follow the merger histories of several candidate dark matter halos from the cosmological simulation that end up within the cluster at later times. We select three halos with circular velocities $\sim 200 \mathrm{~km} \mathrm{~s}^{-1}$ that have suffered very little merging over this period and would therefore be most likely to host disk galaxies. We extract these halos from the simulation at $z=0.5$ and replace the entire halo with the pre-built high resolution model galaxies. We rescale the disk and halo scale lengths by $(1+z)^{-1}$ according to the prescription of Mao et al. (1998) to represent the galaxies entering the cluster at higher redshifts. On a 32 node parallel computer, each run takes several hours; three runs were performed in which the halos were replaced with LSB disks and a further three runs using HSB disks.

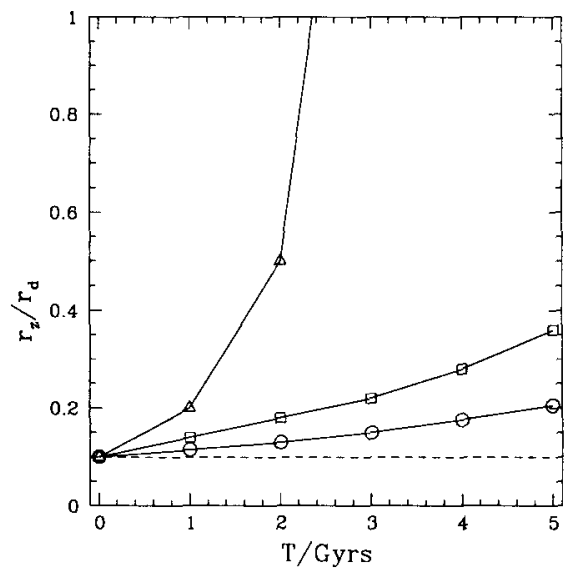

Figure 4. The vertical scale height, $r_{z}$, of the disk in units of the initial disk scale length, $r_{d}$, measure at $r_{d}$ and plotted against time. The circles show the HSB galaxy placed in a void to test the numerical heating. The squares and triangles show one of the HSB and LSB galaxies that enters the cluster respectively. 
At $z=0.5$, the cluster is only just starting to form from a series of mergers of several individual group and galaxy sized halos. The cluster quickly virialises, although several dark matter clumps survive the collapse and remain intact orbiting within the clusters virial radius. Between $z=0.4-0.3$ the model galaxy receives a series of large tidal shocks from the halos that are assembling the cluster. Once the galaxy enters the virialised cluster, it continues to suffer encounters with infalling and orbiting substructure. By a redshift $\mathrm{z}=0.1$, many stars have been stripped from the disk and now orbit through the cluster - closely following the rosette orbit of the parent galaxy. Of the three LSB galaxy runs, between $60 \%$ and $90 \%$ of the stars were harassed from the disk, whereas the stellar mass loss in the HSB runs was between $10 \%-30 \%$.

\section{Summary}

The response of a disk galaxy to tidal shocks is governed primarily by the concentration of the mass distribution that encompasses the visible disk. LSB galaxies have slowly rising rotation curves and dynamical timescales that are constant within their central regions. LSB galaxies cannot survive the chaos of cluster formation; gravitational tidal shocks from the merging substructure literally tear these systems apart, leaving their stars orbiting freely within the cluster and providing the origin of the intra-cluster light.

Recent observations of individual planetary nebulae within clusters, but outside of galaxies, lends support to this scenario. Estimates of the total diffuse light within clusters, using CCD photometry (Bernstein et al. 1995, Tyson \& Fischer 1995) or the statistics of intra-cluster stars (Theuns \& Warren 1997, Feldmeier et al. 1998, Mendez et al. 1998, Ferguson et al. 1998), ranges from $10 \%$ to $45 \%$ of the light attached to galaxies. Presumably, these stars must have originated within galactic systems. The integrated light within LSB galaxies may be equivalent to the light within "normal" spirals (Bothun, Impey \& McGaugh 1997, and references within). This is consistent with the entire diffuse light in clusters originating from harassed LSB galaxies.

High surface brightness disk galaxies and galaxies with luminous bulges have steep mass profiles that give rise to flat rotation curves over their visible extent. The orbital time within a couple of disk scale lengths is short enough for the disk to respond adiabatically to rapid encounters. Tidal shocks cannot remove a large amount of material from these galaxies, nor transform them between morphological types, but will heat the disks and drive instabilities that can funnel gas into the central regions (Lake et al. 1998). A few Gyrs after entering a cluster, their disks are thickened and no spiral features remain. If ram-pressure is efficient at removing gas from disks, we speculate that these galaxies will rapidly evolve into SO's. Since the harassment process and rampressure stripping are both more effective near the cluster centers, we expect that a combination of these effects may drive the morphology-density relation within clusters. 


\section{References}

Bernstein, G.M., Nichol R.C., Tyson J.A., Ulmer M.P. \& Wittman D. 1995, AJ, 110, 1507.

de Blok W.J.G. \& McGaugh S.S. 1996, ApJ, 469, L89.

Bothun G.D., Schombert J.M., Impey C.D., Sprayberry D. \& McGaugh S.S. 1993, AJ, 106, 530.

Butcher, H. and Oemler, A. 1978, ApJ, 219, 18.

Byrd, G. and Valtonen, M. 1990, ApJ, 350, 89.

Couch, W.J., Barger, A.J., Smail, I., Ellis R.S. \& Sharples R.M. 1998, in press.

Dressler, A, Oemler, A., Butcher, H. and Gunn, J.E. 1994a, ApJ, 430, 107.

Dressler A., Oemler A., Couch W.J., Smail I., Ellis R.S., Barger A., Butcher H., Poggianti B.M., Sharples R.M. 1998, ApJ, in press.

Dubinski J., 1998, ApJ, in press.

Feldmeier J, Ciardullo R. \& Jacoby G. 1998, ApJ, in press.

Ferguson H.C., Tanvir N.R. \& von Hippel T. 1998, accepted to Nature (astro$\mathrm{ph} / 9801228)$.

Ghigna, S., Moore, B., Governato, F., Lake, G., Quinn, T. \& Stadel, J. 1998, MNRAS, in press.

Gunn J.E. \& Gott J.R. 1972, ApJ, 176, 1.

Hernquist, L. 1993, ApJS, 86, 389.

Icke, V. 1985, Astr. Ap. 144, 115-23.

Lake, G., Katz, N. and Moore, B. 1998, ApJ, 495, 152.

Lavery R.J. \& Henry J.P. 1988, ApJ, 330, 596.

Lavery R.J. \& Henry J.P. 1994, ApJ, 426, 524.

Mao S., Mo. H.J \& White S.D.M. 1998, MNRASin press.

Mendez, R.H., Guerrero M.A., Freeman K.C., Arnaboldi M., Kudritzki R.P., Hopp U., Capacciolo M. \& Ford H. 1997, ApJ, 491, 23.

Mihos J.C., McGaugh S.S. \& de Blok W.J.G. 1997, ApJ, 477, L79.

Mo H.J., McGaugh S.S. \& Bothun G.D. 1994, MNRAS, 267, 129.

Moore, B., Katz N., Lake G., Dressler, A. and Oemler, A. 1996a, Nature 379, 613.

Moore, B., Katz, N. and Lake, G. 1996b, ApJ, 457, 455.

Moore, B., Lake, G. \& Katz, N. 1998, ApJ, 495, 139.

Persic M. \& Salucci P., 1997, Dark and visible matter in galaxies ASP Conference series, 117 ed. M. Persic P. Salucci.

Theuns T. \& Warren S.J. 1997, MNRAS, 284, L11.

Thompson, L.A. and Gregory, S.A. 1993, AJ, 106, 2197.

Tyson J.A. \& Fischer P. 1995, ApJ, 446, L55.

Valluri, M. and Jog, C. J. 1991, ApJ, 374, 103.

Zwaan M.A., van der Hulst J.M., de Blok W.J.G. \& McGaugh S.S. 1995, MNRAS, 273, L35. 